\title{
Topology Change in Canonical Quantum Cosmology
}

\author{
Vitorio A. De Lorenci ${ }^{(a)}$, Jérôme Martin ${ }^{(a),(b)}$, Nelson Pinto-Neto ${ }^{(a)}$ and \\ Ivano Damião Soares ${ }^{(a)}$
}

(a) Laboratório de Cosmologia e Física Experimental de Altas Energias, Centro Brasileiro de Pesquisas Físicas, Rua Dr. Xavier Sigaud 150, Urca, Rio de Janeiro CEP 22290-180-RJ, Brazil. E-mail: nen@lca1.drp.cbpf.br

(b) Laboratoire de Gravitation et Cosmologies Relativistes, Université Pierre et Marie Curie, CNRS/URA 769, Tour 22/12, Boite courrier 142, 4 place Jussieu, 75252 Paris, France. E-mail:jmartin@ccr.jussieu.fr

\begin{abstract}
We develop the canonical quantization of a midisuperspace model which contains, as a subspace, a minisuperspace constituted of a Friedman-Lemaître-RobertsonWalker Universe filled with homogeneous scalar and dust fields, where the sign of the intrinsic curvature of the spacelike hypersurfaces of homogeneity is not specified, allowing the study of topology change in these hypersurfaces. We solve the Wheeler-DeWitt equation of the midisuperspace model restricted to this minisuperspace subspace in the semi-classical approximation. Adopting the conditional probability interpretation, we find that some of the solutions present change of topology of the homogeneous hypersurfaces. However, this result depends crucially on the interpretation we adopt: using the usual probabilistic interpretation, we find selection rules which forbid some of these topology changes.
\end{abstract}

Pacs numbers: $98.80 . \mathrm{H}, 98.80 . \mathrm{K}$ 


\section{Introduction}

One of the most interesting and fascinating aspects of Quantum Gravity is the idea that the topology of spacetime could change. This question was raised long ago by John Archibald Wheeler in his seminal papers on Quantum Gravity [1], where he described his ideas about the spacetime foam: quantum fluctuations of geometry could enhance quantum changes of topology. In the classical domain, a well-known theorem due to Geroch [2] shows that a generic time orientable manifold endowed with a Lorentzian metric and exhibiting a topology change contains necessarily either closed timelike curves or singularities. If we further assume Einstein's equations, topology changes are forbidden by the unique requirement that spacetime should remain non-singular [3]. Nevertheless, it exists some examples of Lorentzian manifolds presenting a topology change which have only mild singularities (the curvature invariants do not blow up at these points) [4]. As these manifolds have finite Einstein-Hilbert action, they should contribute to the functional integral in the sum-over-histories approach to Quantum Gravity. This fact suggests that topology changes could indeed be possible quantum-mechanically. The aim of this paper is to describe some situations where classically forbidden changes of topology are indeed possible in the quantum domain.

Usually, the articles discussing quantum changes of topology use the path integral formalism and the semi-classical approximation. Some interesting results were obtained in Refs. [5, 6, 7] like, for instance, the proof that, due to the quantum tunneling effect, extremal black holes can be created in pair. Our approach will be based on canonical Quantum Gravity [1, 8], which assumes from the very beginning a topology of the type $R \times V^{3}$. Hence, only space topology changes in $V^{3}$ can be studied in this framework. In this paper, we will consider the following metric:

$$
\mathrm{d} s^{2}=-N^{2}(t) \mathrm{d} t^{2}+a^{2}(t)\left\{\mathrm{d} \chi^{2}+\left[\frac{\sin (\sqrt{k(t)} \chi)}{\sqrt{k(t)}}\right]^{2} \mathrm{~d} \Omega^{2}(\theta, \varphi)\right\}
$$

where $\mathrm{d} \Omega^{2}(\theta, \varphi)$ is the usual line element of a two-sphere. The metric (II) is a FriedmanLemaitre-Robertson-Walker (FLRW) like metric with a time dependent $k(t)$. The idea is that, when $k(t)$ passes through zero, a change of topology occurs $\mathrm{U}$.

However, it is well-known that the metric tensor alone describes a manifold only locally, and does not provide information about its overall structure, i.e., about its topology. Therefore, it is necessary to discuss the features of the metric (11) in more details. In particular, we need to specify the ranges of the variables $(\chi, \theta, \varphi)$.

One of the major tasks of the mathematical research of the nineteenth century was to elaborate a topological classification of spaces. For the purpose of illustration, let us first discuss the two-dimensional case since it is simple (it is sometimes possible to represent a two-dimensional space as a surface embedded in $E^{3}$ ), and since the complete classification is almost entirely known. One can show that any two-dimensional manifold $V^{2}$ is homeomorphic to the quotient $\tilde{V}^{2} / \Gamma$, where $\tilde{V}^{2}$ is the universal covering of $V^{2}$ and $\Gamma$

\footnotetext{
${ }^{1}$ Note that, as the space volume depends on $k(t)$ and $a(t)$, a small $k(t)$ does not necessarily implies a big space volume.
} 
is the group of the covering transformations. The universal covering $\tilde{V}^{2}$ is either $R^{2}$ (the Euclidean plan), $S^{2}$ (the two-sphere) or $H^{2}$ (the hyperbolic plan). In addition the group $\Gamma$ is isomorphic to the fundamental group (or first homotopy group) of $V^{2}: \pi_{1}\left(V^{2}\right)$. Let us consider a simple example: the torus $T^{2}=S^{1} \times S^{1}$. Its universal covering is $R^{2}$ and its first homotopy group is $\pi_{1}\left(T^{2}\right)=Z \oplus Z$. Thus, $T^{2}$ is homeomorphic to $R^{2} /(Z \oplus Z)$. Endowed with this theorem, we see that it is sufficient to know the possible groups $\Gamma$ for being able to classify the two-dimensional manifolds. The classification is complete (except when the universal covering is $H^{2}$ and the manifolds non compact), and can be found, for instance, in Ref. [9].

Let us now consider a more restrictive situation where the surfaces under considerations are closed (here, we use the terminology closed spaces for a compact space without boundary). They are always homeomorphic to a polygon (called the fundamental polyhedron, which is nothing but the development of the largest simply-connected domain of the surface) whose edges are identified by pairs. In this case, one can show that the topology of a surface is uniquely specified by its orientability and the number of its holes (i.e., its genus).

Having at our disposal these mathematical results, we can now ask a slighty different question (but closer to the problem we are interested in): being given a two-dimensional metric, what are the different types of manifolds (i.e., with different topologies) that can be described locally by such a metric? For simplicity, let us consider the metric $\mathrm{d} s^{2}=\mathrm{d} x^{2}+\mathrm{d} y^{2}$. Its scalar curvature is zero. Since, locally, a manifold and its universal covering are indistinguishable, the latter must be $R^{2}$. Then, we can use the previous classification to see that $R^{2}, R \times S^{1}$, the Möbius band, $S^{1} \times S^{1}$ and the Klein bottle, all admit the metric under consideration. If, in addition, we require that the manifold be orientable and closed, the only possibility which remains is $T^{2}=S^{1} \times S^{1}$, as expected. Only the knowledge of some global property, like for instance the ranges of the variables $x$ and $y\left(-L \leq x \leq L,-L \leq y \leq L\right.$ for $T^{2}$, where the opposite edges of the square are identified and $-\infty \leq x \leq+\infty,-\infty \leq y \leq+\infty$ for $R^{2}$ ), can allow us to decide what is the manifold we deal with.

On the other hand, we are obviously not obliged to write the flat metric in the cartesian form. We can also write it with the help of the polar coordinates: $\mathrm{d} s^{2}=\mathrm{d} \chi^{2}+\chi^{2} \mathrm{~d} \theta^{2}$. Then, for the torus $T^{2}$ (we assume here that the fundamental polyhedron is a square whose edge has a length of $2 L$ ), the ranges of the two variables are $0 \leq \theta \leq 2 \pi$ and $0 \leq \chi \leq \chi_{0}(\theta)$, where $\chi_{0}(\theta)=L /|\sin \theta|$ for $\pi / 4 \leq \theta \leq 3 \pi / 4,5 \pi / 4 \leq \theta \leq 7 \pi / 4$ and $\chi_{0}(\theta)=L /|\cos \theta|$ for the other values of $\theta$. The opposite edges are still identified, of course. We would like also to remark that $\chi_{\max } \equiv \max \left\{\chi_{0}(\theta)\right\}=\sqrt{2} L$ is equal to the radius $\chi_{\text {circ }}$ of the circumscribed circle. As a consequence, the area of the torus is less than $\pi \chi_{\max }^{2}=2 \pi L^{2}$, the area of the circumscribed circle. Also, $\chi_{\min } \equiv \min \left\{\chi_{0}(\theta)\right\}=L$ is equal to the radius $\chi_{i n s c}$ of the inscribed circle, and the area of the torus is greater than $\pi L^{2}$, the area of the inscribed circle. Obviously, the value of $\chi_{\text {insc }}, \chi_{\text {circ }}$ depend on the size $L$ of the fundamental polyhedron.

Let us now turn to the three-dimensional case. It actually corresponds to the real situation since we are interested in the topology of the spacelike sections $V^{3}$. More precisely, we would like to answer a question similar to the one already addressed before, 
namely: being given the metric of the three-dimensional hypersurfaces,

$$
\mathrm{d} s^{2}=a^{2}\left\{\mathrm{~d} \chi^{2}+\left[\frac{\sin (\sqrt{k} \chi)}{\sqrt{k}}\right]^{2} \mathrm{~d} \Omega^{2}(\theta, \varphi)\right\},
$$

what are the topologies compatible with such a metric? The techniques described above are still applicable. The scalar curvature of this metric is constant and equal to $6 k / a^{2}$. In addition, we will restrict our considerations to closed (and orientable) spaces in order to avoid possible surface terms in the Hamiltonian formalism. One can show that any compact three-manifold with constant curvature is homeomorphic to $\tilde{V}^{3} / \Gamma$, where the universal covering $\tilde{V}^{3}$ is either $R^{3}, S^{3}$ or $H^{3}$ according to the sign of $k(k=0, k>0$ or $k<0)$. The group $\Gamma$ is still the group of the covering transformations. In three dimensions the closed space is now a polyhedron whose faces are identified by pairs. For a general review see Ref. [10].

Let us first treat the case of a vanishing curvature. It is possible to establish the following result: it exists six closed orientable spaces locally described by the metric (2) with $k=0$. Four of them admit a parallelepiped (in what follows, we will choose a cube) as the fundamental polyhedron, and the two remaining ones an hexagonal prism. Among the first category is the hyper torus $T^{3}=S^{1} \times S^{1} \times S^{1}$. For $T^{3}$, the ranges of the variables $(\chi, \theta, \varphi)$ are given by:

$$
0 \leq \chi \leq \chi_{0}^{(0)}(\theta, \varphi), \quad 0 \leq \theta \leq \pi, \quad 0 \leq \varphi \leq 2 \pi
$$

We will not need to know the explicit form of the function $\chi_{0}^{(0)}(\theta, \varphi)$. The maximum value of $\chi_{0}^{(0)}, \chi_{\max }^{(0)} \equiv \max \left[\chi_{0}^{(0)}(\theta, \varphi)\right]$, which is equal to the radius $\chi_{\text {circ }}$ of the circumscribed sphere, depends on the size of the fundamental cube, which is arbitrary, as it was the case in our two-dimensional example. This would be also true for any other three-manifold with $k=0$. In the remainder of this article, we will work exclusively with $T^{3}$ keeping however in mind that five other choices are possible.

Let us now consider the case $k>0$. The universal covering is $S^{3}$. Since it is compact, all the three-spaces admitting this universal covering are also compact. There are an infinite number of such spaces [11, 12]. We will consider two cases: $S^{3}$ itself and the Poincaré dodecahedral space, $D^{3} \equiv S^{3} / I^{*}$, where $I^{*}$ is the binary symmetry group of the icosahedron. The ranges of $(\chi, \theta, \varphi)$ can be now written as:

$$
0 \leq \chi \leq \chi_{0}^{(1)}\left(\theta, \varphi ; V^{3}\right) / \sqrt{k}, \quad 0 \leq \theta \leq \pi, \quad 0 \leq \varphi \leq 2 \pi
$$

The symbol $V^{3}$ in the argument of $\chi_{0}^{(1)}$ indicates that the function $\chi_{0}^{(1)}\left(\theta, \varphi ; V^{3}\right)$ is not the same whether $V^{3}$ is $S^{3}$ or $D^{3}$. We have $\chi_{0}^{(1)}\left(\theta, \varphi ; S^{3}\right)=\pi$. This guarantees that no conical singularities will appear in this case. If $V^{3}=D^{3}$ the function $\chi_{0}^{(k=1)}$ is more complicated. In this case the maximum value of $\chi_{0}^{(1)}$ is not arbitrary and is given by: $\chi_{\max }^{(1)} \equiv \max \left[\chi_{0}^{(1)}\left(\theta, \varphi ; D^{3}\right)\right] \approx 0.163$.

Finally, we consider the case $k<0$ where the universal covering is $H^{3}$. The classification of hyperbolic closed three-spaces is still an open question. Contrary to the $k=0$ case, the dimensions of the fundamental polyhedron are not arbitrary. Coxeter [9] has 
described fifteen distinct possibilities obtained by tesselation of $H^{3}$. Among them, only four types have their fundamental polyhedron limited. In this article, we will consider the space $I^{3} \equiv H^{3} / \Gamma$ (where $\Gamma$ is an infinite group) studied in Ref. [13]. The ranges of $(\chi, \theta, \varphi)$ are given by:

$$
0 \leq \chi \leq \chi_{0}^{(-1)}(\theta, \varphi) / \sqrt{-k}, \quad 0 \leq \theta \leq \pi, \quad 0 \leq \varphi \leq 2 \pi
$$

The maximum value of $\chi$ can be obtained from the radius of the circumscribed sphere (see above for a comparison with the two-dimensional case), $\chi_{\text {circ }}=\chi_{\max }^{(-1)} \equiv \max \left\{\chi_{0}^{(-1)}(\theta, \varphi)\right\}$, given by $\sinh \chi_{\text {circ }}=\sqrt{\tau^{6} / 4-1} \approx 1.867$ where $\tau \equiv(\sqrt{5}+1) / 2$. Hence, we have $\chi_{\text {max }}^{(-1)} \approx$ 1.382. The minimum value is given by the radius of the inscribed sphere $\chi_{\text {insc }}=\chi_{\text {min }}^{(-1)} \equiv$ $\min \left[\chi_{0}^{(-1)}(\theta, \varphi)\right]$, where $\sinh \chi_{\text {insc }}=\sqrt{3 \tau^{2} / 4-1} \approx 0.982$. This means that $\chi_{\text {min }}^{(-1)} \approx 0.868$.

Let us now come back to the four-dimensional metric (11). Classically, if the matter fields are spatially homogeneous and isotropic, Einstein's equations yield the condition that $k(t)$ must be constant in time. This comes from the momentum constraint equation, $G_{t \chi}=-T_{t \chi}$, which, in this case, is non-trivial giving $[\chi \dot{k}(t)] /[a(t) N(t)]=0$ (a dot means derivation with respect to the time coordinate). Hence, a change of topology becomes impossible ${ }^{2}$. For more complicated matter fields having $T_{t \chi} \neq 0$, the spacetime presents a singularity when $k(t)$ goes to zero. This is because the invariants contain terms like $\dot{k}(t) / k(t)$, which are divergent in the limit where $k(t)$ goes to zero, if $k(t)$ is regular in this vicinity. Our aim is to quantize the model based on Eq. (1) and to study whether or not a transition of the quantum observable associated with $k(t)$ is possible, i.e., if any of the wave functions found can describe, after having adopted a suitable interpretation, a spacetime exhibiting a change of topology. If so, this would demonstrate that topology changes on the hypersurfaces of homogeneity of a FLRW Universe can indeed occur when quantum effects become important.

However, things are not that simple. It is not possible to construct a minisuperspace Hamiltonian from the metric (11) because, as far as $k$ depends on time, this metric does not represent a spatially homogeneous spacetime, in the sense that the components of the four-dimensional curvature tensor in a local frame are functions of $t$ and $\chi$. The existence of the non-null component of the Einstein tensor $R_{t \chi}=[\chi \dot{k}(t)] /[a(t) N(t)] \neq 0$ is a consequence of this fact. Hence we are forced to introduce a midisuperspace model having a non-vanishing shift function $N_{\chi}(\chi, t)$. The metric, which was already proposed in Ref. [14 to study different problems, and also in Ref. [15 to study quantum black holes, can now be written as:

$$
\mathrm{d} s^{2}=\left[-N^{2}(\chi, t)+\frac{N_{\chi}^{2}(\chi, t)}{a^{2}(\chi, t)}\right] \mathrm{d} t^{2}+2 N_{\chi}(\chi, t) \mathrm{d} \chi \mathrm{d} t+a^{2}(\chi, t)\left[\mathrm{d} \chi^{2}+\sigma^{2}(\chi, t) \mathrm{d} \Omega^{2}(\theta, \varphi)\right] .
$$

The first step will be to carry out the quantization of this midisuperspace model. Then, in a second step, we will take into account in the quantum solutions the restrictions on the variables $a$ and $\sigma$, which from (6), allow us to recover the metric (1). We see that

\footnotetext{
${ }^{2}$ Note that, due to the high degree of symmetry of the metric and of the matter fields, the requirements of regularity and causality are not necessary to forbid topology changes in this case. The momentum constraint equation already do the job in a simple way.
} 
consistency requires that we first treat the midisuperspace problem in order to come back to the minisuperspace model afterwards.

This article is organized as follows: in the next section we develop the Lagrangian and Hamiltonian formalism for the midisuperspace model defined by the metric (6). The matter content of the model is described by a dust and a scalar fields $\xi(\chi, t)$ and $\phi(\chi, t)$. In section 3, the quantization of the model is achieved. The dust field is introduced to give a notion of time evolution to the quantum states [16]. We discuss the ordering and anomaly problems, and present some sets of semi-classical solutions to the WheelerDeWitt equation coming from metric (6), restricted to the subspace of the metric (1) and the homogeneous fields $\xi(t), \phi(t)$. In section 4 , we address the issue of interpretation. The dust field permits us to adopt the usual probabilistic interpretation to the wave function of the Universe. We will show that, like a kind of "selection rule", a topology change from $k=1$ and $S^{3}$ topology, to other values of $k$ is not allowed simply because the requirement of normalization of our solutions implies that they must vanish when the topology of $V^{3}$ is $S^{3}$. On the other hand, adopting the conditional probability interpretation, which does not require normalizable wave functions, we will demonstrate that topology changes become possible in this framework even in the case of the $S^{3}$ space. In section 5 , we conclude with comments and perspectives for future works. Finally, the appendix presents other solutions to the quantum equations which, however, are not suitable for the analysis of topology change. We use units such that $c=1$ and $\kappa / 2 \pi=1$, where $\kappa$ is the Einstein's constant.

\section{Hamiltonian Formalism for the Classical Model}

In the Arnowitt-Deser-Misner formalism [17] the Einstein-Hilbert Lagrangean density is given by:

$$
\mathcal{L}_{g}\left[N, N_{i}, \gamma_{i j}\right]=\frac{N \gamma^{\frac{1}{2}}}{4 \pi}\left({ }^{3} R+K^{i j} K_{i j}-K^{2}\right)
$$

where dynamically irrelevant total derivatives have been dropped. In the above expression $\gamma_{i j}$ is the metric of 3 -dimentional spatial sections $t=$ constant. $K_{i j}$ is the extrinsic curvature, $K$ its trace and ${ }^{3} R$ is the scalar curvature of these sections. $N$ and $N_{i}$ are the lapse and shift functions. It will be assumed that the matter content of the model is a minimally coupled scalar field $\phi(\chi, t)$ whose Lagrangian density can be written as:

$$
\mathcal{L}_{\phi}[\phi]=-\frac{\sqrt{-g}}{4 \pi}\left[\frac{1}{2} g^{\alpha \beta} \phi_{, \alpha} \phi_{, \beta}+U(\phi)\right]
$$

and a distribution of irrotational dust particles described by a dust field, see Refs. [16, 18], with Lagrangian density defined by:

$$
\mathcal{L}_{\xi}=-\frac{\sqrt{-g}}{4 \pi}\left[\frac{n}{2 m}\left(g^{\alpha \beta} \xi_{, \alpha} \xi_{, \beta}+m^{2}\right)+V(\xi)\right],
$$

where $m$ is the mass, and $n$ is the rest number density of the dust particles. The dust field $\xi(\chi, t)$ defines the 4 -velocity field for dust particles given by

$$
U^{\mu}=-\frac{g^{\mu \nu} \xi_{, \nu}}{m}
$$


so that $\xi=$ constant will determine a congruence of space-like hypersurfaces foliating the space-time. Therefore, this dust field may be used as the time variable for our model, and in the Schrödinger type equation obtained from the Wheeler-DeWitt equation. Indeed the rest number density $n$ appearing in Eq. (9) has its canonically conjugated momentum $\pi_{n} \approx 0$. Maintaining this constraint demands that $\dot{\xi}=N$ whenever $\xi^{\prime}=0$.

For the class of geometries described by Eq. (6), we define the total Lagrangian density by the expression,

$$
\mathcal{L}=\mathcal{L}_{g}+\mathcal{L}_{\phi}+\mathcal{L}_{\xi}
$$

which takes the following form:

$$
\begin{aligned}
\mathcal{L}= & \frac{a^{3} \sigma^{2} \sin \theta}{4 \pi N}\left\{-6\left(\frac{\dot{a}}{a}\right)^{2}-8\left(\frac{\dot{a} \dot{\sigma}}{a \sigma}\right)-2\left(\frac{\dot{\sigma}}{\sigma}\right)^{2}+4 \frac{\dot{a}}{a^{2}}\left[\left(\frac{N_{\chi}}{a}\right)^{\prime}+\frac{2 N_{\chi}}{a}\left(\frac{a^{\prime}}{a}+\frac{\sigma^{\prime}}{\sigma}\right)\right]\right. \\
& +\frac{4 \dot{\sigma}}{a \sigma}\left[\left(\frac{N_{\chi}}{a}\right)^{\prime}+\frac{N_{\chi}}{a}\left(\frac{a^{\prime}}{a}+\frac{\sigma^{\prime}}{\sigma}\right)\right]-\frac{2 N_{\chi}^{2}}{a^{4}}\left(\frac{a^{\prime}}{a}+\frac{\sigma^{\prime}}{\sigma}\right)^{2}-\frac{4 N_{\chi}}{a^{3}}\left(\frac{N_{\chi}}{a}\right)^{\prime}\left(\frac{a^{\prime}}{a}+\frac{\sigma^{\prime}}{\sigma}\right) \\
& +\frac{\dot{\phi}^{2}}{2}-\frac{N_{\chi} \dot{\phi} \phi^{\prime}}{a^{2}}-N^{2}\left[\frac{\phi^{\prime 2}}{2 a^{2}}+U(\phi)+V(\xi)\right]+\frac{N_{\chi}^{2}}{2 a^{4}} \phi^{\prime 2} \\
& \left.+\frac{n}{2 m}\left[\dot{\xi}^{2}-\frac{2 N_{\chi}}{a^{2}} \dot{\xi}^{\prime}-N^{2}\left(\frac{\xi^{\prime 2}}{a^{2}}+m^{2}\right)+\frac{N_{\chi}^{2} \xi^{\prime 2}}{a^{4}}\right]\right\},
\end{aligned}
$$

where a dot and a prime denote derivative with respect to $t$ and $\chi$, respectively. The total Lagrangian of our model is given by:

$$
L=\int_{V^{3}} \mathrm{~d} \chi \mathrm{d} \theta \mathrm{d} \varphi \mathcal{L}(\chi, t)
$$

If $\chi_{0}(\theta, \varphi)$ does not depend on $(\theta, \varphi)$ (see the discussion above) then the integration of the Lagrangian with respect to these variables can be performed. This amounts to replacing $\sin \theta$ with $4 \pi$ in the expression given by Eq. (12). In that case, it will only remain a single integral over $\chi$ in Eq. (13). Note, however, that for the cases where $\chi_{0}(\theta, \varphi)$ actually depends on $(\theta, \phi)$, the integration over $\theta$ and $\varphi$ cannot be done independently of the integration over $\chi$. The triple integral in Eq. (13) will remain.

Let us now describe the Hamiltonian formalism. We begin with the simplest case where the domain of integration in $\chi$ does not depend on $\theta$ and $\varphi$. The canonical momenta are defined by

$$
\pi_{Q_{i}} \equiv \frac{\delta L}{\delta \dot{Q}_{i}}, \quad \text { for } \quad Q_{i} \equiv\{a, \sigma, \phi, \xi\} .
$$

Using the Lagrangian $L$ given by Eq. (13) and (12), and performing the integration on the $\theta$ and $\varphi$, we easily find that:

$$
\begin{aligned}
& \pi_{a}=\frac{4}{N}\left(-3 a \dot{a} \sigma^{2}-2 a^{2} \sigma \dot{\sigma}+2 \sigma \sigma^{\prime} N_{\chi}+\sigma^{2} N_{\chi}^{\prime}\right) \\
& \pi_{\sigma}=\frac{4 a}{N \sigma}\left(-2 a \dot{a} \sigma-a^{2} \dot{\sigma}+\sigma^{\prime} N_{\chi}+\sigma N_{\chi}^{\prime}\right)
\end{aligned}
$$




$$
\begin{aligned}
\pi_{\phi} & =\frac{a^{3} \sigma^{2}}{N}\left(\dot{\phi}-\frac{N_{\chi}}{a^{2}} \phi^{\prime}\right), \\
\pi_{\xi} & =\frac{n a^{3} \sigma^{2}}{m N}\left(\dot{\xi}-\frac{N_{\chi} \xi^{\prime}}{a^{2}}\right) .
\end{aligned}
$$

The total Hamiltonian density can be calculated by performing the Legendre transformation $\mathcal{H}=\sum_{i} \dot{Q}_{i} \pi_{Q_{i}}-\mathcal{L}$, yielding the following expression:

$$
\mathcal{H}=N \mathcal{H}^{0}+N_{\chi} \mathcal{H}^{\chi}
$$

where $\mathcal{H}^{0}$ and $\mathcal{H}^{\chi}$ are the super-Hamiltonian and super-momentum constraints respectively, and are given by the following equations:

$$
\begin{aligned}
\mathcal{H}^{0} & =\left[\frac{\pi_{a}^{2}}{8 a \sigma^{2}}+\frac{3 \pi_{\sigma}^{2}}{8 a^{3}}-\frac{\pi_{a} \pi_{\sigma}}{2 a^{2} \sigma}+\frac{\pi_{\phi}^{2}}{2 a^{3} \sigma^{2}}\right]+\sigma^{2} a^{3} \mathcal{V}+\sqrt{\frac{\xi^{\prime 2}}{a^{2}}+m^{2}} \pi_{\xi}, \\
\mathcal{H}^{\chi} & =\frac{1}{a^{2}}\left(-a \pi_{a}^{\prime}+\sigma \pi_{\sigma}^{\prime}+2 \sigma^{\prime} \pi_{\sigma}+\phi^{\prime} \pi_{\phi}+\xi^{\prime} \pi_{\xi}\right)
\end{aligned}
$$

the "superpotential" $\mathcal{V}$ being defined by the formula:

$$
\mathcal{V}=-{ }^{3} R+\frac{\phi^{\prime 2}}{2 a^{2}}+U(\phi)+V(\xi)
$$

Here it is worth remarking that Eqs. (19)-(21) can be derived directly from the Hamiltonian $\mathcal{H}=\dot{\gamma}_{i j} \pi^{i j}+\dot{\phi} \pi_{\phi}+\dot{\xi} \pi_{\xi}-\mathcal{L}$, by calculating

$$
\pi_{i j}=\frac{\delta \mathcal{L}}{\delta \dot{\gamma}_{i j}}=\frac{\partial \mathcal{L}}{\partial \dot{a}} \frac{\delta \dot{a}}{\delta \dot{\gamma}_{i j}}+\frac{\partial \mathcal{L}}{\partial \dot{\sigma}} \frac{\delta \dot{\sigma}}{\delta \dot{\gamma}_{i j}} .
$$

for the class of geometries described by Eq. (6).

Also, the Poisson Brackets $(\mathrm{PB})$ for the variables $\left(Q_{i}, \pi_{Q_{i}}\right)$, are given by

$$
\left\{Q_{i}(\chi, t), \pi_{Q_{j}}(\bar{\chi}, t)\right\}=\delta_{i j} \delta(\chi-\bar{\chi}),
$$

all others being zero. We can check that the PB (24) are consistently derived from the standard PB of the ADM variables $\left(\gamma_{i j}, \pi^{i j}\right)$, and that the Hamiltonian (19) provides the correct Einstein's equations for our model.

Before proceeding in the quantization of the above system, it will prove useful to perform a change to new variables $\alpha(\chi, t)$ and $\beta(\chi, t)$ defined by

$$
\alpha=\ln a, \quad \beta=-2 \ln (\sigma a)
$$

The momenta $\pi_{\alpha}$ and $\pi_{\beta}$ are related to the old momenta $\pi_{a}$ and $\pi_{\sigma}$ by

$$
\begin{aligned}
& \pi_{a}=e^{-\alpha}\left(\pi_{\alpha}-2 \pi_{\beta}\right), \\
& \pi_{\sigma}=-2 e^{\alpha+\frac{\beta}{2}} \pi_{\beta} .
\end{aligned}
$$


In the new variables $\alpha$ and $\beta$, the super-Hamiltonian and super-momentum constraints can be expressed as

$$
\begin{aligned}
\mathcal{H}^{0} & =e^{-\alpha+\beta}\left(\frac{\pi_{\alpha}^{2}}{8}+\frac{\pi_{\alpha} \pi_{\beta}}{2}+\frac{\pi_{\phi}^{2}}{2}\right)+e^{\alpha-\beta} \mathcal{V}+\sqrt{e^{-2 \alpha \xi^{\prime 2}+m^{2}}} \pi_{\xi}, \\
\mathcal{H}^{\chi} & =e^{-2 \alpha}\left(\alpha^{\prime} \pi_{\alpha}+\beta^{\prime} \pi_{\beta}+\phi^{\prime} \pi_{\phi}+\xi^{\prime} \pi_{\xi}-\pi_{\alpha}^{\prime}\right)
\end{aligned}
$$

where the functions $\mathcal{V}$ and ${ }^{3} R$ in the $\alpha, \beta$ variables have now the form:

$$
\begin{aligned}
\mathcal{V} & =-{ }^{3} R+e^{-2 \alpha} \frac{\phi^{\prime 2}}{2}+U(\phi)+V(\xi), \\
{ }^{3} R & =e^{-2 \alpha}\left(-\frac{3}{2} \beta^{\prime 2}-2 \alpha^{\prime} \beta^{\prime}+2 \beta^{\prime \prime}+2 e^{2 \alpha+\beta}\right) .
\end{aligned}
$$

Finally, for further reference we also introduce the two variables $u(\chi, t)$ and $v(\chi, t)$ given by:

$$
u \equiv \ln \sigma=-\alpha-\frac{\beta}{2}, \quad v \equiv \ln \left(a^{3} \sigma^{2}\right)=\alpha-\beta .
$$

We remark that $v$ is nothing but the logarithm of the volume density $\gamma^{\frac{1}{2}}$ divided by $\sin \theta$. In these variables the super-Hamiltonian constraint is expressed as:

$$
\begin{aligned}
\mathcal{H}^{0}= & e^{-v}\left[\frac{3}{8}\left(\pi_{u}^{2}-\pi_{v}^{2}\right)+\frac{1}{2} \pi_{\phi}^{2}\right]+\sqrt{e^{\frac{4 u}{3}-\frac{2 v}{3}} \xi^{\prime 2}+m^{2}} \pi_{\xi} \\
& +e^{v}\left[-{ }^{3} R+e^{\frac{4 u}{3}+\frac{2 v}{3}} \frac{\phi^{\prime 2}}{2}+U(\phi)+V(\xi)\right],
\end{aligned}
$$

exhibiting explicitly the Klein-Gordon character of the gravitational sector.

For the cases where $0 \leq \chi \leq \chi_{0}(\theta, \varphi)$, the integration on the $(\theta, \varphi)$ variables cannot be done as previously. In these cases, the Hamiltonian which yields the Einstein's equations is

$$
H=\frac{1}{4 \pi} \int_{0}^{2 \pi} \mathrm{d} \varphi \int_{0}^{\pi} \mathrm{d} \theta \sin \theta \int_{0}^{\chi_{0}(\theta, \varphi)} \mathrm{d} \chi\left[N(\chi, t) \mathcal{H}^{0}(\chi, t)+N_{\chi}(\chi, t) \mathcal{H}^{\chi}(\chi, t)\right],
$$

where $\mathcal{H}^{0}(\chi, t)$ and $\mathcal{H}^{\chi}(\chi, t)$ are exactly the same as the ones given in Eqs. (20) and (21), or in Eqs. (28) and (29). The momenta also given by Eqs. (15)-(18) and the Poisson brackets are the same as (24). The constraints are still given by:

$$
\begin{gathered}
\frac{\delta H}{\delta N(\chi, t)}=0 \Rightarrow \mathcal{H}^{0}(\chi, t)=0, \\
\frac{\delta H}{\delta N_{\chi}(\chi, t)}=0 \Rightarrow \mathcal{H}^{\chi}(\chi, t)=0 .
\end{gathered}
$$

We would like to emphasize that the Hamiltonian (34) furnishes the correct Einstein's equations for whatever function $\chi_{0}(\theta, \varphi)^{3}$.

Let us now turn to the quantization of the classical model described above.

\footnotetext{
${ }^{3}$ The Hamiltonian density in Eq. (34) may be obtained directly from the Langrangian "density" $\overline{\mathcal{L}}=4 \pi \mathcal{L} / \sin \theta$. The quantity $\overline{\mathcal{L}}$ is still a density with respect to transformations involving only the $\chi$ variable. One should remember that the fields depend only on $\chi$.
} 


\section{Quantization}

In order to quantize the constrained system described in the last section, we follow the Dirac's prescriptions [19]. The variables of the phase space go into operators acting on the wave functional $\Psi[a, \sigma, \phi, \xi]$, the Poisson brackets turn into commutators and the momentum operators are represented by:

$$
\hat{\pi}_{Q_{i}} \equiv-i \hbar \frac{\delta}{\delta Q_{i}}, \text { for } \quad Q_{i} \equiv\{a, \sigma, \phi, \xi\}
$$

Also, the space of the wave functionals must be endowed with the structure of a Hilbert space in order to associate operators with observables. Finally, the super-Hamiltonian and super-momentum constraints given by Eqs. (28), (29), (35) and (36) result in two relations which express that only a restricted region of the Hilbert space of the wave functionals contains the physical states of the theory:

$$
\hat{\mathcal{H}}^{0} \Psi[a, \sigma, \phi, \xi]=0, \quad \hat{\mathcal{H}}^{\chi} \Psi[a, \sigma, \phi, \xi]=0 .
$$

The first equation is the well-known Wheeler-DeWitt equation, whereas the second one is the so-called momentum constraint equation. In the remainder of this paper, we will be mainly interested in solutions of these equations. Note that the formulas (38) are valid for all possible domains of variation of $\chi$, and thus applicable to all possible topologies we may consider.

At this point, it should be emphasized that the knowledge of the wave functional alone does not allow us to compute probabilities and/or mean values unless a measure has been specified. In order to address this question we notice that the Wheeler-De Witt Hamiltonian (33) with the following factor ordering:

$$
\begin{aligned}
\hat{\mathcal{H}}^{0}= & \frac{3}{8}\left[\hat{\pi}_{u} e^{-v} \hat{\pi}_{u}-\hat{\pi}_{v} e^{-v} \hat{\pi}_{v}\right]+\frac{1}{2} \hat{\pi}_{\phi} e^{-v} \hat{\pi}_{\phi}+\sqrt{m^{2}+e^{\frac{2}{3}(2 u-v)} \xi^{\prime 2}} \hat{\pi}_{\xi} \\
& +e^{v}\left[-{ }^{3} R+\frac{1}{2} e^{\frac{2}{3}(2 u-v)} \phi^{\prime 2}+U(\phi)+V(\xi)\right] \\
= & \frac{3}{8} e^{-v}\left[\hat{\pi}_{u}^{2}-\hat{\pi}_{v}^{2}-i \hbar \hat{\pi}_{v} \delta(0)\right]+\frac{e^{-v}}{2} \hat{\pi}_{\phi}^{2}+\sqrt{m^{2}+e^{\frac{2}{3}(2 u-v)} \xi^{\prime 2}} \hat{\pi}_{\xi} \\
& +e^{v}\left[-{ }^{3} R+\frac{e^{\frac{2}{3}(2 u-v)}}{2} \phi^{\prime 2}+V(\phi)+U(\chi)\right],
\end{aligned}
$$

is hermitian if one chooses the "cartesian" measure defined by:

$$
\mathcal{D} u \mathcal{D} v \mathcal{D} \phi
$$

Note that $\hat{\pi}_{\xi}$ appears linearly in the Hamiltonian constraint (40) allowing to interpret the dust field $\xi$ as a time variable at the quantum level. This is why $\mathcal{D} \xi$ does not appear in the measure (41). In the remainder of this article we will adopt this measure.

We would like to stress that the anomaly problem is still present. Indeed, strictly speaking, we should check that the algebra of the constraints is preserved at the quantum level. However, in the following, we will be dealing exclusively with WKB solutions valid 
at the $\hbar^{0}$ order and this problem will not be important for us. Also the solutions will be independent of the factor ordering chosen previously.

The super-Hamiltonian given by Eq. (33) can be re-expressed in terms of the variables $\alpha$ and $\beta$. We obtain the following expression for $\hat{\mathcal{H}}^{0}$ :

$$
\begin{aligned}
\hat{\mathcal{H}}^{0}= & {\left[\frac{\hat{\pi}_{\alpha} e^{-\alpha+\beta} \hat{\pi}_{\alpha}}{8}+\frac{1}{4}\left(\hat{\pi}_{\alpha} e^{-\alpha+\beta} \hat{\pi}_{\beta}+\hat{\pi}_{\beta} e^{-\alpha+\beta} \hat{\pi}_{\alpha}\right)+\frac{\hat{\pi}_{\phi}^{2}}{2}\right]+e^{\alpha-\beta} \mathcal{V} } \\
& +\sqrt{e^{-2 \alpha}{\xi^{\prime}}^{2}+m^{2}} \hat{\pi}_{\xi} .
\end{aligned}
$$

As announced previously, the equation $\hat{\mathcal{H}}^{0} \Psi=0$ is a Schrödinger like functional equation with $\xi$ playing the role of time. This Hamiltonian is obviously hermitian in the cartesian measure $\mathcal{D} \alpha \mathcal{D} \beta \mathcal{D} \phi$ and can be also re-written as:

$$
\begin{aligned}
\hat{\mathcal{H}}^{0}= & e^{-\alpha+\beta}\left[\frac{\hat{\pi}_{\alpha}^{2}}{8}+\frac{\hat{\pi}_{\alpha} \hat{\pi}_{\beta}}{2}+\frac{\hat{\pi}_{\phi}^{2}}{2}\right]+i \hbar e^{-\alpha+\beta}\left(\frac{3 \hat{\pi}_{\alpha}}{8}-\frac{\hat{\pi}_{\beta}}{4}\right) \delta(0)+e^{\alpha-\beta} \mathcal{V} \\
& +\sqrt{e^{-2 \alpha \xi^{\prime 2}}+m^{2}} \hat{\pi}_{\xi} .
\end{aligned}
$$

Let us now turn to the solutions of Eqs. (38). A general wave functional can always be put under the following form:

$$
\Psi[\alpha, \beta, \phi, \xi]=e^{\frac{i}{\hbar} \mathcal{S}[\alpha, \beta, \phi, \xi]},
$$

where the functional $\mathcal{S}[\alpha, \beta, \phi, \xi]$ can be complex. If we introduce the form (44) into the Wheeler-De Witt equation and the momentum constraint, we find that $\mathcal{S}$ must satisfy the equations:

$$
\begin{aligned}
& -i \hbar e^{-\alpha+\beta}\left[\frac{1}{8} \frac{\delta^{2} \mathcal{S}}{\delta \alpha^{2}}+\frac{1}{2} \frac{\delta^{2} \mathcal{S}}{\delta \alpha \delta \beta}+\frac{1}{2} \frac{\delta^{2} \mathcal{S}}{\delta \phi^{2}}-\left(\frac{3}{8} \frac{\delta \mathcal{S}}{\delta \alpha}-\frac{1}{4} \frac{\delta \mathcal{S}}{\delta \beta}\right) \delta(0)\right]+ \\
& e^{-\alpha+\beta}\left[\frac{1}{8}\left(\frac{\delta \mathcal{S}}{\delta \alpha}\right)^{2}+\frac{1}{2}\left(\frac{\delta \mathcal{S}}{\delta \alpha}\right)\left(\frac{\delta \mathcal{S}}{\delta \beta}\right)+\frac{1}{2}\left(\frac{\delta \mathcal{S}}{\delta \phi}\right)^{2}\right]+e^{\alpha-\beta} \mathcal{V}+\sqrt{e^{-2 \alpha \xi^{\prime 2}+m^{2}}} \frac{\delta \mathcal{S}}{\delta \xi}=0 \\
& e^{-2 \alpha}\left[\alpha^{\prime} \frac{\delta \mathcal{S}}{\delta \alpha}+\beta^{\prime} \frac{\delta \mathcal{S}}{\delta \beta}+\phi^{\prime} \frac{\delta \mathcal{S}}{\delta \phi}+\xi^{\prime} \frac{\delta \mathcal{S}}{\delta \xi}-\partial_{\chi}\left(\frac{\delta \mathcal{S}}{\delta \alpha}\right)\right]=0
\end{aligned}
$$

The factor ordering in the momentum constraint (46) is the natural one because it implies that the wave functional depends only on the 3-geometry of the sections $t=$ constant. At order $\hbar^{0}$, Eq. (45) reduces to the Hamilton-Jacobi (H-J) functional equation, whose solution is the phase of the WKB wave functional. The momentum constraint equation (46) remains unchanged.

As it has already been noticed, a topology change in our model can be studied only in a midisuperspace framework because one is forced to introduce a shift function in order to produce the correct Einstein's equations. This is technically more difficult because we now deal with equations which are no longer ordinary differential equations but functional differential equations. It is clear that Eqs. (45) and (46) are very complicated. We were not able to find solutions valid in the full midisuperspace $M$. The solutions we obtained 
are only valid in the minisuperspace $\mathcal{M}$, i.e., they are solutions in the subdomain $\mathcal{M} \subset M$

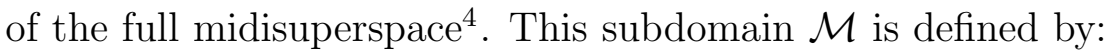

$$
a^{\prime}=0, \quad \phi^{\prime}=0, \quad \xi^{\prime}=0, \quad \sigma=\frac{\sin (\sqrt{k(t)} \chi)}{\sqrt{k(t)}} .
$$

Under these assumptions several solutions to the complete functional equations (45) and (46) (i.e., in $\mathcal{M}$ ) can be obtained. However, they are not suitable for the study of topology change. We present some of them in the appendix. In the following we restrict ourselves to WKB solutions. In this case, it is possible to find solutions where we can examine the possibility of a topology change that may happen quantum-mechanically. For $U(\phi)=V(\xi)=0$, we display two solutions to both the H-J and the momentum constraint functional equations

$$
\begin{aligned}
& \mathcal{S}_{1}=\frac{1}{4 \pi} \int_{V^{3}} \mathrm{~d} \tau\left\{-\frac{C}{m}\left(\beta^{\prime} e^{-\alpha-\beta}\right)^{\prime} \xi+\beta^{\prime} e^{-\beta}\left[\sqrt{\frac{3-C}{2}} \phi+\sqrt{6+2 C}\left(\alpha-\ln \left(-\beta^{\prime}\right)\right)\right]\right\} \\
& \mathcal{S}_{2}=\frac{1}{4 \pi} \int_{V^{3}} \mathrm{~d} \tau\left\{-\frac{C}{m}\left(\beta^{\prime} e^{-\alpha-\beta}\right)^{\prime} \xi+4 i \sqrt{C+3} e^{\alpha-\frac{\beta}{2}}+i \sqrt{2 C+3} \beta^{\prime} \phi e^{-\beta}\right\}
\end{aligned}
$$

where $\mathrm{d} \tau=\mathrm{d} \chi \mathrm{d} \varphi \mathrm{d} \theta \sin \theta$ and $C$ is an arbitrary constant. It is worth emphasizing that they are solutions to the momentum constraint equation in the full midisuperspace $M$. In order to check that these solutions satisfy the H-J functional equation, we must first perform the functional derivatives, then substitute them into the H-J equation, and finally make the restriction to $\mathcal{M}$, namely, take into account the conditions (47). Note that if we use the classical relations $\pi_{Q_{i}}=\delta \mathcal{S} / \delta Q_{i}$ where the $\pi_{Q_{i}}$ are given by Eqs. (15), (16), (17) and (18), we re-obtain the result $\dot{k}=0$.

The last step is to perform the integration with respect to the variable $\chi$. This leads to the following wave functions:

$$
\begin{aligned}
\Psi_{1}= & \exp \left(\frac{i \mathcal{S}_{1}}{\hbar}\right) \\
= & \exp \left\{\frac { i } { 4 \pi \hbar } \int _ { 0 } ^ { 2 \pi } \mathrm { d } \varphi \int _ { 0 } ^ { \pi } \mathrm { d } \theta \operatorname { s i n } \theta \left[\frac{C}{m} \frac{a}{\sqrt{k}} \xi \sin \left(2 \lambda_{0}\right)-\sqrt{\frac{3-C}{2}} \phi \frac{a^{2}}{k} \sin ^{2} \lambda_{0}\right.\right. \\
& \left.\left.-\sqrt{6+2 C} \frac{a^{2}}{k}\left(\sin ^{2} \lambda_{0} \ln \left(\frac{a}{2 \sqrt{k}} \sin \lambda_{0}\right)+\cos \lambda_{0} \ln \left(\cos \lambda_{0}\right)\right)\right]\right\} \\
\Psi_{2}= & \exp \left(\frac{i \mathcal{S}_{2}}{\hbar}\right) \\
= & \exp \left\{\frac { i } { 4 \pi \hbar } \int _ { 0 } ^ { 2 \pi } \mathrm { d } \varphi \int _ { 0 } ^ { \pi } \mathrm { d } \theta \operatorname { s i n } \theta \left[\frac{C}{m} \frac{a}{\sqrt{k}} \xi \sin \left(2 \lambda_{0}\right)-i \sqrt{2 C+3} \phi \frac{a^{2}}{k} \sin ^{2} \lambda_{0}\right.\right. \\
& \left.\left.+8 i \sqrt{C+3} \frac{a^{2}}{k} \sin ^{2}\left(\frac{\lambda_{0}}{2}\right)\right]\right\},
\end{aligned}
$$

\footnotetext{
4 For instance, this is similar to obtaining solutions of a partial differential equation, $\left[\frac{\partial}{\partial x^{2}}+\frac{\partial}{\partial y^{2}}-\frac{\partial}{\partial t^{2}}+V(x, y, t)\right] f(x, y, t)=0$, restricted to a spatial subdomain, say $y=0$.
} 
where $\lambda_{0} \equiv \sqrt{\epsilon} \chi_{0}^{(\epsilon)}(\theta, \varphi)$ and where the symbol $\epsilon \equiv \operatorname{sign}(k)$ is defined by $\epsilon=0$ if $k=0$ and $\epsilon= \pm 1$ if $k>0$ or $k<0$. The above expressions are valid whatever the sign of $k$ is. For instance, if $k<0$ the term proportional to $\xi$ becomes $(a / \sqrt{-|k|}) \sin \left[2 i \chi_{0}^{(-1)}(\theta, \varphi)\right]=$ $(a / \sqrt{|k|}) \sinh \left[2 \chi_{0}^{(-1)}(\theta, \varphi)\right]$. For $k=0$, this term would be equal to $2 a \chi_{0}^{(0)}(\theta, \varphi)$.

On the other hand, under the change of coordinates $\bar{\chi}=\sqrt{k(t)} \chi, \bar{\theta}=\theta, \bar{\varphi}=\varphi$, the line element of the three-dimensional spacelike sections, see Eq. (2), transforms into the following expression:

$$
\mathrm{d} s^{2}=\frac{a^{2}(t)}{k(t)}\left[\mathrm{d} \bar{\chi}^{2}+\sin ^{2}(\bar{\chi}) \mathrm{d} \Omega^{2}(\theta, \varphi)\right] .
$$

We see that the metrics $\{k(t)>0, a(t)\}$ and $\{1, \bar{a}(t)=a(t) / \sqrt{k(t)}\}$ are simply related by a diffeomorphism. Equivalently, this is also true for the metrics $\{k(t)<0, a(t)\}$ and $\{-1, \bar{a}(t)=a(t) / \sqrt{-k(t)}\}$. In other words, they belong to the same equivalence class, and describe the same three-geometry in the minisuperspace, i.e., they are represented by a "single point" in $\mathcal{M}$. We remark that the wave functions $\Psi_{1}$ and $\Psi_{2}$ depend only on the combination $a(t) / \sqrt{|k|}$, which can be redefined as a new scale factor $\bar{a}(t)=a(t) / \sqrt{|k|}$. This is exactly what was expected since $\Psi_{1}$ and $\Psi_{2}$ are solutions to the momentum-constraint equation and therefore must be invariant under three-dimensional diffeomorphisms on $V^{3}$. The wave functions $\Psi_{1}$ and $\Psi_{2}$ depend only on $\epsilon$ and not on $k$.

Let us also emphasize that our procedure for obtaining the wave functions for $k=0, \pm 1$ is by no mean equivalent to the "naive" procedure which would consist in constructing a minisuperspace for each value of $k$ and in solving the corresponding Wheeler-DeWitt equation in each case. Indeed, in our model the dynamical behaviour of $k(t)$ is encoded in the momentum $\pi_{\sigma}$ which does not appear at all in the naive approach where $k$ is fixed. It is well-known that in this case the gravitational sector of the Wheeler-De Witt equation contains only $\pi_{a}$. It is easy to see that the presence of this term modifies drastically the problem. The naive approach is therefore completely different from the formalism considered here.

The above wave functions $\Psi_{1}$ and $\Psi_{2}$ are the main result of this section. In the next paragraph, we will try to extract physical information from them.

\section{Interpretation}

\subsection{Standard Probabilistic Interpretation}

In this section, we turn to the problem of the interpretation of the wave function. Conceptually, this question is one of the most difficult challenges one faces in trying to construct a consistent theory of Quantum Gravity. In General Relativity, the principle of covariance is expressed through the diffeomorphism invariance which leads to the constraints. After quantization à la Dirac, those constraints transform into functional differential equations for the state $\Psi$. The momentum constraint indicates that $\Psi$ depends only on the three-geometry of the spacelike sections and therefore is a functional on superspace. The Hamiltonian constraint expresses the fact that $\Psi$ is independent of the time variable occurring in the Hamiltonian formalism. This fact is understandable since $\Psi$ is supposed 
to describe the Universe as a whole and therefore, by definition, cannot depend on some exterior parameter. This causes severe problems when one tries to interpret Quantum Gravity because time plays a fundamental role in the structure of Quantum Mechanics 20 .

The situation described above seems to be similar to what happens in the parametrized version of non-relativistic particle mechanics. In this theory, the path followed by the particle can be parametrized by a label $\tau$, and the absolute Newtonian time $t$ can be considered as one of the dynamical variables. After quantization, this approach leads to an equation $H \Psi=0$ comparable to the Wheeler-DeWitt equation. However, in this case, there exists a criterion which allows one to identify immediately what is the time variable: the Hamiltonian is linear in the momentum canonically conjugated to $t$, whereas it is quadratic in the momenta conjugated to the space variables. Hence, the equation $H \Psi=0$ reduces to the ordinary Schrödinger equation.

In Quantum Gravity the situation is different. The Hamiltonian constraint, i.e., the Wheeler-DeWitt equation, is quadratic in all the momenta, and a priori we have no mean to recognize what is time in this context. However, the idea to introduce a Hamiltonian which is linear in one of the momenta has been pursued by many authors. The extrinsic time approach advocated by Teitelboim and Kuchař [21, 22] is one of these proposals. It consists in performing a canonical transformation such that the Wheeler-De Witt Hamiltonian becomes linear in the momentum conjugated to the trace of the extrinsic curvature $K$ (which should be viewed as time). However, the corresponding Schrödinger Hamiltonian is known only through the solution of a complicated differential equation. Therefore, this approach remains unsatisfactory.

Another proposal has been made by Kuchař and Torre [16]. The idea is to introduce a dust field $\xi\left(t, x^{i}\right)$ as one of the sources of matter because the Hamiltonian is first order in $\pi_{\xi}$. This was our main motivation for having considered $\xi\left(t, x^{i}\right)$ in the previous sections. Even if the interpretation of this dust field in the very early Universe remains rather obscure, it gives us a well-defined structure where we can ask physical relevant questions. As a matter of fact, limitations will come only from technical problems.

In a first step, we will try to use this framework in order to interpret the solutions of section 3, and to see if they describe a spacetime which displays a change of topology.

It is clear that the most efficient method would be to compute the quantum propagator defined by:

$$
K\left(a_{i}, \sigma_{i}, \phi_{i}, \xi_{i} \mid a_{f}, \sigma_{f}, \phi_{f}, \xi_{f}\right)=\int \mathcal{D} a \mathcal{D} \sigma \mathcal{D} \phi \mu(a, \sigma, \phi) e^{\frac{i}{\hbar} S[a, k, \phi, \xi]}
$$

where $\mu(a, \sigma, \phi)$ is the measure. Then, expressing this propagator for the function $\sigma(t, \chi)$ given in Eq. (47) we would be able to determine the probability of having $k_{f}=1$, for instance, at "time" $\xi_{f}$ knowing that $k_{i}$ was equal to -1 (or 0 ) at initial time $\xi_{i}$. A non vanishing result would demonstrate explicitly the possibility of a change of topology at the quantum level. Unfortunately, computing the propagator (53) is technically very complicated. Another possibility would be to evaluate $K\left(a_{i}, \sigma_{i}, \phi_{i}, \xi_{i} \mid a_{f}, \sigma_{f}, \phi_{f}, \xi_{f}\right)$ at the semi-classical level using the well-known result of Van Vleck and Gutzwiller [23, 24]. However, this would require the knowledge of all the classical trajectories.

Having only at our disposal the states given by Eqs. (50) and (51), we can also try to compute the mean value of different interesting quantities. For example, a good candidate 
would be the three-curvature:

$$
{ }^{3} R>\equiv<\left.\Psi\right|^{3} R \mid \Psi>
$$

Another natural possibility would be the operator $-\sigma^{\prime \prime} / \sigma$ which, in the minisuperspace $\mathcal{M}$ where the solutions (50)-(51) are valid, reduces simply to $k$ :

$$
<k>\equiv<\Psi\left|-\frac{\sigma^{\prime \prime}}{\sigma}\right| \Psi>\left.\right|_{\mathcal{M}} .
$$

In what follows, we will restrict our considerations to the calculation of Eq. (55) in $\mathcal{M}$. Then, we will be able to study the sign of $k$ as a function of $\xi$, the "time" in our system.

It has been emphasized above that, in order to compute mean values and norm of wave functionals, a measure should be chosen. In the previous section, we adopted the cartesian measure $\mathcal{D} \alpha \mathcal{D} \beta \mathcal{D} \phi$. Therefore, after having put the Wheeler-DeWitt equation in the form of a Schrödinger equation, the solutions should be normalized according to the formula:

$$
<\Psi \mid \Psi>=\int_{M} \mathcal{D} \alpha \mathcal{D} \beta \mathcal{D} \phi \Psi[\alpha, \beta, \phi, \xi] \Psi^{*}[\alpha, \beta, \phi, \xi] .
$$

The fact that the Hamiltonian, with the cartesian measure, is hermitian automatically guarantees that $\langle\Psi \mid \Psi\rangle$ will not depend on $\xi$. In practice computing the integral (56) is a difficult task. In addition, this requires the knowledge of the wave functional in the full midisuperspace $M$, whereas we know $\Psi$ only in the minisuperspace $\mathcal{M} \subset M$. Endowed with these solutions, we can only write a necessary but not sufficient condition in order to have a normalized wave functional, namely:

$$
<\Psi \mid \Psi>_{\mathcal{M}} \equiv \int_{\mathcal{M} \subset M} \mathcal{D} \alpha \mathcal{D} \beta \mathcal{D} \phi \Psi[\alpha, \beta, \phi, \xi] \Psi^{*}[\alpha, \beta, \phi, \xi]<\infty
$$

This equation can be worked out. The ranges of $\phi$ and $\xi$ are given by $-\infty<\phi<+\infty$ and $-\infty<\xi<+\infty$. The domain of $a$ is $(0,+\infty)$, and from the discussion of the previous section we see that integrating over $k$ will amount to sum over $k \in\{0, \pm 1\}$. The result is that $\langle\Psi \mid \Psi\rangle_{\mathcal{M}}$ can be put under the form:

$$
\begin{aligned}
<\Psi \mid \Psi>_{\mathcal{M}}= & \int_{-\infty}^{\infty} \mathrm{d} \alpha \sum_{k=0, \pm 1} \int_{-\infty}^{\infty} \mathrm{d} \phi \int_{M} \mathcal{D} \alpha \mathcal{D} \beta \mathcal{D} \phi \delta[\alpha(t, \chi)-\alpha(t)] \\
& \delta\left\{\beta(t, \chi)+\ln \left[\frac{k(t)}{a^{2} \sin ^{2}(\sqrt{k(t)} \chi)}\right]\right\} \delta[\phi(t, \chi)-\phi(t)] \\
& \Psi[\alpha, \beta, \phi, \xi] \Psi^{*}[\alpha, \beta, \phi, \xi] \\
= & \sum_{k=0, \pm 1} \int_{-\infty}^{\infty} \mathrm{d} \alpha \int_{-\infty}^{\infty} \mathrm{d} \phi \Psi(\alpha, k, \phi, \xi) \Psi^{*}(\alpha, k, \phi, \xi),
\end{aligned}
$$

where $\delta[f-g]$ is the $\delta$-functional defined by the expression (see Ref. [25):

$$
\delta[f-g] \equiv \prod_{\chi} \delta(f(\chi)-g(\chi)) .
$$


Finally, since $\alpha \equiv \ln a$, we obtain the following condition:

$$
<\Psi \mid \Psi>_{\mathcal{M}}=\sum_{k=0, \pm 1} \int_{0}^{+\infty} \frac{\mathrm{d} a}{a} \int_{-\infty}^{\infty} \mathrm{d} \phi \Psi(a, k, \phi, \xi) \Psi^{*}(a, k, \phi, \xi)<\infty
$$

Let us now come back to our solutions $\Psi_{1}$ and $\Psi_{2}$, which we will denote by $\Psi_{i}, i=$ 1,2. They depend on an arbitrary complex constant $C$. Therefore, the principle of superposition implies that the most general state satisfying the Wheeler-DeWitt equation which we can construct with a single state $\Psi_{i}$, is given by:

$$
\Psi_{i}(a, k, \phi, \xi)=\int_{\mathcal{C}} \mathrm{d} C f_{i}(C) e^{\frac{i}{\hbar} S_{i}(C ; a, k, \phi, \xi)},
$$

where $f_{i}(C)$ is an arbitrary function. Of course, we could also construct wave functions which would be superpositions of the states $\Psi_{1}$ and $\Psi_{2}$. The function $f_{i}(C)$ should be chosen such that $\Psi$ be normalized. Let us fix the $S^{3}$ topology for $k=1$. It follows that $0 \leq \chi \leq \pi$ and $S_{i}(C ; a, k=1, \phi, \xi)=0$ for any value of $C$ [see Eqs. (50) and (51)]. Hence, the states $\Psi_{i}$ reduces to:

$$
\Psi_{i}(a, k=1, \phi, \xi)=\int_{\mathcal{M}} \mathrm{d} C f_{i}(C) .
$$

Therefore, in the sum over $k$ in Eq. (61), the contribution coming from the term $k=1$ is equal to:

$$
\int \mathrm{d} C \mathrm{~d} C^{\prime} f_{i}(C) f_{i}^{*}\left(C^{\prime}\right) \int_{0}^{+\infty} \frac{\mathrm{d} a}{a} \int_{-\infty}^{\infty} \mathrm{d} \phi
$$

and the only way to obtain a normalized wave function is to choose $f_{i}(C)$ such that:

$$
\left|\int \mathrm{d} C f_{i}(C)\right|^{2}=0 .
$$

From Eq. (63) we see that $\Psi_{i}(k=1)=0$. Note that this condition is a necessary condition in order to obtain a normalized wave functional extended from the solutions $\Psi_{1}$ and $\Psi_{2}$ to the full midisuperspace $M$. Of course, $f_{i}(C)$ must also be taken such that the two other contributions coming from the terms $k=0$ and $k=-1$ in the sum (61) be finite.

Let us now turn to the computation of the mean value of $k$. By definition, the expression for $<k>$ can be written as:

$$
\begin{aligned}
<k>(\xi) & =\sum_{k=0, \pm 1} \int_{0}^{+\infty} \frac{\mathrm{d} a}{a} \int_{-\infty}^{\infty} \mathrm{d} \phi \Psi(a, k, \phi, \xi) k \Psi^{*}(a, k, \phi, \xi) \\
& =-\int_{0}^{+\infty} \frac{\mathrm{d} a}{a} \int_{-\infty}^{\infty} \mathrm{d} \phi \Psi(a, k=-1, \phi, \xi) \Psi^{*}(a, k=-1, \phi, \xi),
\end{aligned}
$$

where the last formula was obtained using the property (65). From Eq. (67) we deduce that:

$$
<k>(\xi) \leq 0
$$

We remark that this result is rigorous in the sense that it has been established from considerations valid in the full midisuperspace $M$. Equation (68) tells us that the change 
of topology from $S^{3}$ to $k=0$ or $k=-1$ is forbidden whereas the passage from $k=0$ to $k=-1$, or the opposite only for the solutions presented in section 3, assuming the $S^{3}$ topology for $k=1$, and at the $\hbar^{0}$ order. It is interesting to note that, already at this level, the different possibilities are not equivalent. In order to demonstrate that a change of topology from $k=0$ to $k=-1$ is actually possible, an explicit function $f_{i}(C)$ should be considered. Also, if we had chosen the $D^{3}$ topology for $k=1$, then $0 \leq \chi \leq \chi_{0}^{(1)}(\theta, \varphi)$ and $S_{i}(C ; a, k=1, \phi, \xi)$ would no longer be a vanishing quantity. Hence, it would no longer be necessary to have $\Psi_{i}(k=1)=0$ in order to have a normalizable wave function. In principle, topology change from $k=1$ and a topology different from $S^{3}$ could be possible in the standard probabilistic interpretation. However, we leave these questions open and turn now to a different scheme of interpretation.

\subsection{The Conditional Probability Interpretation}

The result of the previous section crucially depends on our ability to normalize the wave function. However, it has been recognized long time ago that unnormalized wave functions can also be used to make predictions if one utilizes them to compute conditional probabilities. This approach has been advocated by many authors in the context of minisuperspaces, where the wave function is in general not normalizable. In the case of a FLRW spacetime filled with a scalar field, this allows one to compute the probability distribution for the initial value of the scalar field [26]. Here, we will use this formalism in order to learn something about $k$.

Suppose we have found that a spacelike hypersurface is homogeneous and isotropic, with the scale factor given by some value $a=\bar{a}$, and the scalar field given by $\phi=\bar{\phi}$. Then, the conditional probability of having this spacelike hypersurface with $k$ equal to $-1,0$ or +1 , at some "time" $\xi$, knowing that $a=\bar{a}$ and $\phi=\bar{\phi}$, will be given by:

$$
P_{c}(k \mid \bar{a}, \bar{\phi}) \equiv \frac{|\Psi(k, \bar{a}, \bar{\phi})|^{2}}{\sum_{k=0, \pm 1}|\Psi(k, \bar{a}, \bar{\phi})|^{2}} .
$$

The constraint $\sum_{k=0, \pm 1} P_{c}(k \mid \bar{a}, \bar{\phi})=1$ can be immediately checked. Let us compute and discuss $P_{c}(k \mid \bar{a}, \bar{\phi})$ for the wave function $\Psi_{2}$. At this stage, boundary conditions on $\Psi_{2}$ should be chosen. In the present context, this amounts to fix the value of the constant $C$ [for the solution (62) this would amount to choose the function $f_{i}(C)$ and the contour $\mathcal{C}$ ]. Many proposals have been made to answer this question [27, 28]. We will consider two different possible values for $C$ in order to illustrate that different choices actually lead to different predictions. Here, our aim is just to exhibit at least one state for which the change of topology considered in this article is possible, and it should be clear that the two wave functions $\Psi_{1}$ and $\Psi_{2}$ do not represent automatically states of cosmological interest.

As a first example, let us assume that $C=-i$. The term multiplying $\xi$ in the

\footnotetext{
${ }^{5}$ It would be interesting to know if it is more likely to have a transition from $k=0$ to $k=-1$ or from $k=-1$ to $k=0$. In the framework developed here, this information can not be given
} 
exponential of Eq. (51) becomes:

$$
\begin{aligned}
F_{1}\left(V^{3}\right) & \equiv \frac{\bar{a}}{4 \pi \hbar m} \int_{0}^{2 \pi} \mathrm{d} \varphi \int_{0}^{\pi} \mathrm{d} \theta \sin \theta \sin \left[2 \chi_{0}^{(1)}\left(\theta, \varphi ; V^{3}\right)\right], \quad \text { if } \quad k=1, \\
F_{0}\left(T^{3}\right) & \equiv \frac{\bar{a}}{4 \pi \hbar m} \int_{0}^{2 \pi} \mathrm{d} \varphi \int_{0}^{\pi} \mathrm{d} \theta \sin \theta\left[2 \chi_{0}^{(0)}(\theta, \varphi)\right], \quad \text { if } \quad k=0, \\
F_{-1}\left(I^{3}\right) & \equiv \frac{\bar{a}}{4 \pi \hbar m} \int_{0}^{2 \pi} \mathrm{d} \varphi \int_{0}^{\pi} \mathrm{d} \theta \sin \theta \sinh \left[2 \chi_{0}^{(-1)}(\theta, \varphi)\right], \quad \text { if } \quad k=-1 .
\end{aligned}
$$

The numerical values on the $F_{i}$ 's determine the behaviour of the conditional probabilities. If $V^{3}=S^{3}$, then $F_{1}\left(S^{3}\right)=0$. For the other cases it is not possible to calculate the $F_{i}$ 's exactly because we do not know the form of $\chi_{0}^{(\epsilon)}(\theta, \varphi)$. But we can put bounds on their numerical values, and it will be sufficient for our purpose here. Let us start with $F_{1}\left(D^{3}\right)$. We know (see the Introduction) that $\chi_{\max }^{(1)}=0.163$. For the interval of integration under consideration, the integrand in Eq. (70) is positive. This implies that:

$$
F_{1}\left(D^{3}\right) \leq \frac{\bar{a}}{\hbar m} \sin [0.326] \approx 0.320 \frac{\bar{a}}{\hbar m}
$$

In the same manner, when $k$ is negative, we can establish that:

$$
2.75 \frac{\bar{a}}{\hbar m} \leq F_{-1}\left(I^{3}\right) \leq 7.90 \frac{\bar{a}}{\hbar m} .
$$

Note that $F_{-1}$ is always greater than $F_{1}$. The case $k=0$ is slighty different. As pointed out before, the value of $\chi_{\max }^{(0)}$ depends on the fundamental cube of $T^{3}$, which is arbitrary. Hence, $F_{0}$ is also arbitrary.

Using the definition (69) and Eq. (51), we obtain the following expressions for the conditional probabilities:

$$
\begin{aligned}
P_{c}(k=-1) & =\frac{A(\bar{a}, \bar{\phi}) e^{2 F_{-1} \xi}}{A(\bar{a}, \bar{\phi}) e^{2 F_{-1} \xi}+B(\bar{a}, \bar{\phi}) e^{2 F_{0} \xi}+C(\bar{a}, \bar{\phi}) e^{2 F_{1} \xi}}, \\
P_{c}(k=0) & =\frac{B(\bar{a}, \bar{\phi}) e^{2 F_{0} \xi}}{A(\bar{a}, \bar{\phi}) e^{2 F_{-1} \xi}+B(\bar{a}, \bar{\phi}) e^{2 F_{0} \xi}+C(\bar{a}, \bar{\phi}) e^{2 F_{1} \xi}}, \\
P_{c}(k=+1) & =\frac{C(\bar{a}, \bar{\phi}) e^{2 F_{1} \xi}}{A(\bar{a}, \bar{\phi}) e^{2 F_{-1} \xi}+B(\bar{a}, \bar{\phi}) e^{2 F_{0} \xi}+C(\bar{a}, \bar{\phi}) e^{2 F_{1} \xi}},
\end{aligned}
$$

where the positive functions $A(\bar{a}, \bar{\phi}), B(\bar{a}, \bar{\phi})$ and $C(\bar{a}, \bar{\phi})$ can be calculated from Eq. (51). Since we will be interested in the behaviour of $P_{c}(k)$ at the boundaries of the minisuperspace in $\xi$, these functions will not be relevant for what follows because they do not depend on $\xi$. For clarity, we will treat the two possibilities for $k=1$ considered in this article, separately.

If $V^{3}=S^{3}$, then $F_{1}=0$ and we have two possibilities: $F_{0}>F_{-1}$ or $F_{0}<F_{-1}$. From the previous expressions, we get:

$$
\lim _{\xi \rightarrow-\infty} P_{c}(k=-1)=\lim _{\xi \rightarrow-\infty} P_{c}(k=0)=0, \quad \text { and } \quad \lim _{\xi \rightarrow-\infty} P_{c}(k=+1)=1 .
$$


On the other hand, if we compute the same probabilities but this time when the dust field goes to $+\infty$, we obtain:

$$
\begin{aligned}
\lim _{\xi \rightarrow+\infty} P_{c}(k=+1) & =0, \\
\lim _{\xi \rightarrow+\infty} P_{c}(k=-1) & =\left\{\begin{array}{lll}
0 & \text { if } & F_{0}>F_{-1}, \\
1 & \text { if } & F_{0}<F_{-1},
\end{array}\right. \\
\lim _{\xi \rightarrow+\infty} P_{c}(k=0) & =\left\{\begin{array}{lll}
0 & \text { if } & F_{0}<F_{-1}, \\
1 & \text { if } & F_{0}>F_{-1} .
\end{array}\right.
\end{aligned}
$$

Therefore, in this case, definite predictions can be made since conditional probabilities are either equal to 0 or 1 . If $F_{0}<F_{-1}$ there is a change of topology from $k=1$ to $k=-1$ when we go from $\xi=-\infty$ to $\xi=+\infty$ in the minisuperspace. On the other hand, if $F_{0}>F_{-1}$, there is a change of topology from $k=1$ to $k=0$. It is clear that this is a quantum mechanical effect since it is not possible that a classical path could connect the two regions. A similar result would have been obtained if we had used $\Psi_{1}$ instead of $\Psi_{2}$.

For the set $\left\{D^{3}, T^{3}, I^{3}\right\}$, we have $0<F_{1}<F_{-1}$ and three possibilities:

a) $F_{0}<F_{1}$. The conditional probabilities (75)-( 77 give for $\xi \rightarrow-\infty$

$$
P_{c}(k=0)=1
$$

and for $\xi \rightarrow+\infty$

$$
P_{c}(k=-1)=1 ;
$$

b) $F_{1}<F_{0}<F_{-1}$. For $\xi \rightarrow-\infty$ we have

$$
P_{c}(k=+1)=1
$$

and for $\xi \rightarrow+\infty$,

$$
P_{c}(k=-1)=1 ;
$$

c) $F_{1}<F_{-1}<F_{0}$. For $\xi \rightarrow-\infty$ we have

$$
P_{c}(k=+1)=1
$$

and for $\xi \rightarrow+\infty$,

$$
P_{c}(k=0)=1 \text {. }
$$

It is interesting to notice that the behaviour of the conditional probabilities for the choice $C=i$ can be obtained directly from the previous results (obtained for $C=-i$ ) just by replacing $\xi$ by $-\xi$. This shows the importance of the choice of boundary conditions on the final result.

At this point, we would like to make an important remark. The conditional probabilities exhibited before possess an interesting property. It seems that it exists "privileged directions" for the change of topology. For $C=-i$, for instance, it always occurs from the smallest $F_{i}$ to the biggest $F_{j}$. Note that the $F_{i}$ are as big as it is the volume of the closed hypersurfaces they are referred to. Whether this property is generic or just a consequence of the particular approximated solutions found here is, of course, a question which remains to be solved. 
Let us conclude this section by emphasizing that different interpretations actually lead to very different results. For instance, the "selection rule" established previously is violated in the conditional probability interpretation. This shows how crucial the problem of the interpretation of Quantum Gravity is.

\section{Conclusion}

In this article, we have addressed the problem of topology change in canonical Quantum Cosmology. More precisely, we have treated the question of whether a quantum change of topology can occur between two spacelike hypersurfaces of a FLRW metric whose intrinsic curvatures have a different sign.

A consistent Hamiltonian treatment of this problem requires an enlargement of the minisuperspace model to a midisuperspace model. This enlarged configuration space is, per se, very interesting to analyze because the corresponding theory contains an infinite number of degrees of freedom and is therefore a (Quantum) Field Theory, closer to the full Quantum Gravity than the usual minisuperspace models. New problems arise, like anomalies and regularization, and the Wheeler-DeWitt equation becomes a functional differential equation. We have also seen that the physical predictions depend crucially on the interpretation we adopt. In the standard probabilistic interpretation, we have shown that the changes of topology $S^{3}(k=+1) \rightarrow T^{3}(k=0)$ or $S^{3}(k=+1) \rightarrow I^{3}(k=-1)$ are forbidden for the particular set of solutions we have obtained. On the other hand, if we apply the conditional probability interpretation to this same set of solutions, a wide class of quantum changes of topology are actually possible, including the ones forbidden by the standard probabilistic interpretation mentioned above.

There are many perspectives for future works. One could try to find an exact normalizable solution (with the measure defined in section 3) in the full midisuperspace $M$, and try to use the probabilistic interpretation in order to check if topology changes can occur in this case. In fact, exact solutions to the Hamilton-Jacobi equations (45) and (46) already exist in the literature but only with $\phi=\xi=0$ 15 or with $\xi=0$ and a dilaton field [29]. It should be very interesting to study if these solutions can predict a topology change ${ }^{6}$. New families of solutions involving only the more fundamental scalar field could also be obtained by mean of the the long-wavelength approximation [18]. In this case, topology changes should be examined between hypersurfaces characterized by different values of the scale factor.

In this paper, we have restricted ourselves to the study of the spaces $\left\{S^{3}, D^{3}, T^{3}, I^{3}\right\}$. As pointed out in the Introduction, many other cases are a priori possible. Therefore, one could calculate the corresponding conditional probabilities in order to see if new topological transitions are possible. Note that even open spaces with $k=0$ could be also considered because the surface terms which could appear in the Hamiltonian formalism vanish in this case.

It would be interesting to calculate the probability amplitude for having change of topology of the surfaces of homogeneity by using the path integral approach. To our

\footnotetext{
${ }^{6}$ Also, it would be worthwhile to examine if the predictions given by these midisuperspace solutions agree with the ones known in the literature for the minisuperspace models they contain, see Ref. 30].
} 
knowledge, this approach has never been applied to this particular problem. In principle one should find a solution of the classical equations in the Euclidean midisuperspace which, when reduced to the minisuperspace subspace, could be interpreted as an instanton connecting two homogeneous hypersurfaces with distinct topologies. The exponential of the Euclidean action evaluated at this solution would then give the semi-classical probability amplitude for change of topology. To find such a solution is, however, a very difficult task.

We hope to address these questions in future publications.

\section{Acknowledgments}

We are grateful to G. Clément, H. Fagundes, L. Ford, R. Kerner, B. Linet, V. Moncrief, P. Spindel, A. F. da F. Teixeira and the group of the "Pequeno Seminário" for useful discussions. R. Portugal is acknowledged by providing us with his maple program "RIEMANN". We thank CNPq for financial support.

\section{Appendix}

In this appendix, we present solutions to the Wheeler-DeWitt and momentum constraint equations which are different from those already found in Eqs. (48) and (49). All of them are solutions restricted to the minisuperspace $\mathcal{M}$. Although they are of limited interest for the problem of topology change, they could be compared to the solutions directly obtained from the minisuperspace formulation. In the variables $(\alpha, \beta)$ they can be written as:

$$
\Psi=\exp \left(\frac{i}{\hbar} \mathcal{S}[\alpha, \beta, \xi, \phi]\right)
$$

A first class of solutions is valid only for the case ${ }^{3} R=0$. Obviously, they do not allow a change of topology. An example is given by:

$$
\mathcal{S}[\alpha, \beta, \phi]=\frac{1}{4 \pi} \int_{V^{3}} \mathrm{~d} \tau\left[e^{\alpha-\beta} e^{-\frac{9 \beta}{4} \pm \sqrt{3} \phi}\right] .
$$

These wave functionals are solutions to the momentum constraint equation (46) and the full Wheeler-DeWitt equation (45) with ${ }^{3} R=0$.

A second class of solutions solves the functional equations (45) and (46) for ${ }^{3} R \neq 0$. They are given by:

$$
\begin{aligned}
\mathcal{S}[\alpha, \beta, \xi, \phi] & =\frac{1}{4 \pi} \int_{V^{3}} \mathrm{~d} \tau\left[-3\left(\beta^{\prime} e^{-\alpha-\beta}\right)^{\prime} \xi+C e^{\alpha-\frac{\beta}{4}}+\sqrt{3} \beta^{\prime} \phi e^{-\beta}\right], \\
\mathcal{S}[\alpha, \beta, \phi] & =\frac{1}{4 \pi} \int_{V^{3}} \mathrm{~d} \tau \beta^{\prime} e^{-\beta}\left[\sqrt{\frac{3}{2}} \phi+\sqrt{6}\left(\alpha-\ln \left(-\beta^{\prime}\right)\right)\right],
\end{aligned}
$$

where $C$ is an arbitrary constant. We note that, for both Eqs. (84) and (85), the corresponding wave functions lead to "time-independent" conditional probabilities.

We would like to remark that the last functional (85) is an exact solution to the Wheeler-DeWitt functional equation restricted to $\mathcal{M}$, while (84) is a WKB solution to Eq. (45). 


\section{References}

[1] J. A. Wheeler, Ann. Phys. 2, 604 (1957) and in Relativity, Groups and Topology, ed. by B. DeWitt and C. DeWitt (Gordon and Breah, New York, 1964).

[2] R. Geroch, J. Math. Phys. 8, 782 (1967).

[3] F. Tipler, Ann. Phys. 108, 1 (1977).

[4] G. T. Horowitz, Class. Quantum Grav. 8, 587 (1991).

[5] S. Coleman, Nucl. Phys. B307, 864 (1988).

[6] S. Giddings and A. Strominger, Nucl. Phys. B306, 890 (1988); Nucl. Phys. B307, 854 (1988).

[7] F. Dowker, J. P. Gauntlett, S. B. Giddings, and G. T. Horowitz, Phys. Rev. D50, 2662 (1994).

[8] B. S. DeWitt, Phys. Rev. 160, 1113 (1967).

[9] H. S. M. Coxeter, Twelve Geometric Essays, (Southern Illinois University Press, 1968).

[10] M. Lachièze-Rey and J. P. Luminet, Phys. Report 254, 135 (1995)

[11] H. Seifert and W. Threlfall, Math. Ann. 104, 1 (1930); A textbook of Topology edited by J. S. Birman and J. Eisner, (New York: Academic, 1980).

[12] H. Poincaré, Oeuvres de Henri Poincaré, Vol. VI, (Gauthier-Villars, Paris, 1953); I. N. Bernshtein and F. V. Shvartsman, Sov. Phys. JETP 52, 814 (1980).

[13] L. A. Best, Can. J. Math. 23, 451 (1971); H. Fagundes, Ap. J. 338, 618 (1989).

[14] W. Fischler, D. Morgan and J. Polchinski, Phys. Rev. D42, 4042 (1990).

[15] T. Brotz and C. Kiefer, Report gr-qc/9608031.

[16] K. V. Kuchař and C. G. Torre, Phys. Rev. D43, 419 (1991).

[17] R. Arnowitt, S. Deser, and C. W. Misner, in Gravitation: An Introduction to Current Research, edited by L. Witten (Wiley, New York, 1962).

[18] D. S. Salopek, Phys. Rev. D46, 4373 (1992).

[19] P. A. M. Dirac, Lectures on Quantum Mechanics (Yeshiva University, New York, 1964); M. Henneaux and C. Teitelboim, Quantization of Gauge Systems (Princeton University Press, Princeton, 1992); K. Sundermeyer, Constrained Dynamics (Springer-Verlag, Berlin, 1982).

[20] W. G. Unhru and R. M. Wald, Phys. Rev. D40, 2598 (1984). 
[21] C. Teitelboim, Phys. Lett. B56, 376 (1975).

[22] K. Kuchar, in Quantum Gravity II edited by C. J. Isham, R. Penrose and D. W. Sciama, (Clarendon Press, Oxford, 1982).

[23] J. H. Van Vleck, Proc. Natl. Acad. Sci. 14 (1928) 178.

[24] M. C. Gutzwiller, Chaos in Classical and Quantum Mechanics (Springer-Verlag, Berlin, 1990).

[25] B. Hatfield, "Quantum Field Theory of Point Particles and Strings", (AddisonWesley, Redwood City, 1992).

[26] J.J. Halliwell, in Quantum Cosmology and Baby Universes edited by S. Coleman, J. B. Hartle, T. Piran and S. Weinberg, (World Scientific, Singapore, 1991).

[27] J. B. Hartle and S. W. Hawking, Phys. Rev. D28, 2960 (1983).

[28] A. Vilenkin, Phys. Rev. D37, 888 (1988).

[29] D. Louis-Martinez at al, Phys. Lett. B321, 193 (1994); J. G. Demers and C. Kiefer, Phys. Rev. D53, 7050 (1996).

[30] K. V. Kuchar and M. P. Ryan, Phys. Rev. D40, 3982 (1989). 